\title{
Urgences
}

\section{Section inter du domaine}

\section{Renald Bérubé et André Gervais}

Numéro 19, janvier 1988

Le tour du texte

URI : https://id.erudit.org/iderudit/025444ar

DOI : https://doi.org/10.7202/025444ar

Aller au sommaire du numéro

Éditeur(s)

Urgences

ISSN

0226-9554 (imprimé)

1927-3924 (numérique)

Découvrir la revue

Citer ce document

Bérubé, R. \& Gervais, A. (1988). C. Section inter du domaine. Urgences, (19),

39-51. https://doi.org/10.7202/025444ar d'utilisation que vous pouvez consulter en ligne.

https://apropos.erudit.org/fr/usagers/politique-dutilisation/ 


\section{Section inter du domaine:}

intertextuation

intertexte, intertextualité

téléintratexte: voir intertexte

infra-biblio-intertexte

ultra-biblio-intertexte

graphotexte

anthologicotexte

bibliotexte

infra-inter-bibliotexte

ultra-inter-bibliotexte

syntexte

péritexte

hypotexte

pluritextuel: voir hypotexte hypertexte, hypertextualité contretexte

mimotexte, mimotextualité

intermimotexte, intermimotextualité 


\section{Intertextuation}

RÉF. - Michel van Schendel, 1986-1987

DÉF. - "Je résume. $L$ * intertexte est donné à lire par l'intertextuation. Lintertextuation est un acte de lecture singularisé par un acte d'écriture, par la * textuation d'un texte singulier."

Michel van Schendel: "Liidéologème est un quasi-argument», Texte. Toronto, no 5/6 (no intitule Théories du texte), 1986-1987, p. 120.

\section{Intertexte, intertextualité}

RÉF. - Julia Kristeva, 1966; Jean Ricardou, 1974 et 1983; Michaël Riffaterre, 1979; Gérard Genette, 1982

DEFF. - «En introduisant la notion de statut du mot comme unité minimale de la structure, Bakhtine situe le texte dans l'histoire et la société, envisagées elles-mêmes comme textes que l'écrivain lit et dans lesquels il s'insère en les récrivant. [...] le mot (le texte) est un croisement de mots (de textes) où on lit au moins un autre mot (texte). (...Cette] découverte que Bakhtine est le premier à introduire dans la théorie littéraire: tout texte se construit comme mosaïque de citations, tout texte est absorption et transformation d'un autre texte. À la place de la notion d'intersubjectivité s'installe celle d'intertextualité, et le langage poétique se lit, au moins, comme double.» Julia Kristeva: Sèméiotikè. Recherches pour une sémanalyse. Coll. "Tel Quel», Paris, Seuil, 1969, p. 144-146.

> > On aura compris, à la lecture de ce montage d'extraits, que J. Kristeva, utilisant "texte" là où $M$. Bakhtine utilise "mot», fait naître, en quelque sorte sous nos yeux, le terme «intertextualité».

$\$$ «Dans l'étude présentée au colloque Claude Simon de Cerisy-laSalle (1974), une discrimination est établie entre intertextualité générale (rapports intertextuels entre textes d'auteurs différents) et intertextualité restreinte (rapports intertextuels entre textes du même auteur). Or cette démarcation n'est pas superposable à celle que propose Pour une théorie du nouveau roman (1971) où, soucieux de mettre en cause l'unité d'une oeuvre et la notion corrélative d'Auteur. Ricardou en arrivait fort logiquement à distinguer entre une intertextualité externe entendue comme rapport diun texte à un autre texte et une intertextualité interne comprise comme rapport d'un texte à lui-même.

II n'entre pas dans nos intentions d'harmoniser deux systèmes 
qui, sans doute, ne peuvent exister que concurremment."

Lucien Dällenbach: «Intertexte et autotexte». Poétique. Paris, no 27, 1976, p. 282

>> Selon toute vraisemblance, la distinction établie en 1974 (et dont la référence exacte est Jean Ricardou: " "Claude Simon" textuellement". Claude Simon/Colloque de Cerisy (colloque tenu en 1974l. Coll. "10/18", no 945, Paris, UGE, 1975, p. 10-11) a mieux résisté que celle suggérée en 1971 , puisque l'un des deux termes de cette dernière, l' «intertextualité interne», est désormais remplacé par le terme * intratexte.

>> L'«inter-texte» selon J. Bellemin-Noël (Le texte et l'avant-texte, p. 138) est l' «intertexte restreint" selon J. Ricardou.

>> Le téléintratexte selon A. Gervais (La raie alitée d'effets. Apropos of Marcel Duchamp. Coll. "Brèches", Montréal, Hurtubise HMH, 1984 , p. 8), c'est-à-dire les rapports en quelque sorte télécommandés entre les textes qui sont aux extrémités d'une oeuvre, souvent à plusieurs dizaines d'années de distance, est une variante de précision de l'«intertexte restreint» selon J. Ricardou.

+ "Ce que, sous de soigneuses précautions, un intertexte apporte à un texte, c'est son appui dans les deux phases distinctes du mème combat. D'une part, selon l'alliance intertextuelle. vis-à-vis du commun adversaire, il ajoute un surcroit de liens qui peuvent excéder l'expression ou la représentation. Alors, il se joint à la force textuelle dans le combat antireprésentatif. D'autre part, selon le conflit intertextuel, interne aux deux alliés, il interdit au texte de se prendre à l'ultime piège de son identité prétendue. Alors, il apporte à la force textuelle une arme contre l'éventuel retour insidieux, en elle, au second degré, de la représentation.

[...]

Admettre, selon les voeux que j'ai fait miens, lintertextualité comme "l'aptitude pour tel élément d'un texte à se mettre en.rapport avec un ou plusieurs éléments d'un autre texte», ce n'est en rien pressentir la structure de l'intertexte. Or, celle-ci oscille entre deux modèles contraires. L'un pourrait se nommer intertexte scalène. Ce qui le compose? D'une part, un texte récepteur, objet principal de l'écriture ou de la lecture. D'autre part, au moins un texte donneur, auquel, disons, la greffe citationnelle est soustraite. C'est cette structure inégalitaire qu'illustrent, chacun à sa manière, les exemples offerts un peu plus haut. L'autre type pourrait s'appeler intertexte paritaire. Ce qui le constitue? Un ensemble localisé de textes où aucun ne joue, en pivot, spécialement le rôle de récepteur. C'est une structure égalitaire de cet ordre, s'agissant du Nouveau Roman, que le colloque (de Cerisyl de 1971 a construite." 
Jean Ricardou: «Le texte survit à l'excité (Réponse à Michel Holland)». Texte. Toronto, no 2 (no intitulé L intertextualité. Intertexte, autotexte, intratexte), 1983, p. 201 et 205.

EX. — L'article ici cité analyse (p. 198-199 et 201-206) ces exemples.

$\S$ «ll est nécessaire de préciser la définition, parce que la critique actuelle se contente trop souvent d'appeler intertexte l'ensemble des oeuvres qu'un lecteur peut rapprocher de celui qu'il a sous les yeux, l'ensemble des passages que lui rappelle tel morceau qui lui plait (et qui ne lui plait, souvent, que parce qu'il lui rappelle quelque chose). Ainsi compris, l'intertexte varie selon le lecteur: les passages que celui-ci réunit dans sa mémoire, les rapprochements qu il fait, lui sont dictés par l'accident d'une culture plus ou moins profonde plutôt que par la lettre du texte. Je crois au contraire que les fluctuations de l'intertexte ne relèvent pas du hasard, mais de structures, dans la mesure où une structure admet des variantes plus ou moins nombreuses, mais qui ramènent toutes à un invariant. L'intertexte, quelle que soit son étendue pour un lecteur donné a des éléments constants, entièrement réglés par des impératifs textuels. [...]

[...] il y a intertextualité obligatoire, lorsque la signification de certains mots du texte n'est ni celle que permet la langue ni celle que demande le ${ }^{*}$ contexte, mais le sens qu'ont ces mots dans l'intertexte. C'est l'inacceptabilité de ce sens dans la langue ou dans le * contexte qui contraint le lecteur à une présomption, à l'hypothèse d'une solution offerte dans un homologue formel du texte quil essaie de déchiffrer. L'intertexte, par conséquent, n'est pas un objet de citation, c'est un objet présupposé.

[... Le transfert de signifiance entre deux oeuvres est lui-même surdéterminé par la présence latente de systèmes de signes qui médiatisent la référence du texte à son intertexte. Ces systèmes médiatisants sont eux aussi des intertextes; mais leur fonction est spécialisée: ils se comportent comme des interprétants, au sens qu'a le terme en sémiotique (peirciennel. (...)

La signifiance, c'est-à-dire la littérarité du sens, n’est donc ni dans le texte ni dans l'intertexte, mais à mi-chemin des deux, dans l'interprétant, qui dicte au lecteur la manière de les voir, de les comparer, de les interpréter par conséquent dans leur inséparabilité même."

Michaël Riffaterre: "La trace de l'intertexte". La pensée. Paris, no 215 (no intitulé Approches actuelles de la littérature), octobre 1980, p. 4-5, 7 et 14-15.

EX. - L'article ici cité analyse l'intertexte de textes de J. Derrida, S. Mallarmé et $\mathrm{G}$. de Nerval.

$\S$ «Le premier [type de relations * transtextuelles] a été, voici quelques années, exploré par Julia Kristeva, sous le nom d'intertextualité, et 
cette nomination nous fournit évidemment notre paradigme terminologique. Je le définis pour ma part d'une manière sans doute restrictive, par une relation de coprésence entre deux ou plusieurs textes, c'est-à-dire, eidétiquement et le plus souvent, par la présence effective d'un texte dans un autre. Sous sa forme la plus explicite et la plus littérale, c'est la pratique traditionnelle de la citation (avec' guillemets, avec ou sans référence précise); sous une forme moins explicite et moins canonique, celle du plagiat (chez Lautréamont, par exemple), qui est un emprunt non déclaré, mais encore littéral; sous forme encore moins explicite, celle de l'allusion, c'est-à-dire d'un énoncé dont la pleine intelligence suppose la perception d'un rapport entre lui et un autre auquel renvoie nécessairement telle ou telle de ses inflexions, autrement non recevables. (...) Cet état implicite (et parfois tout hypothétique) de l'intertexte est depuis quelques années le champ d'étude privilégié de Michaël Riffaterre, qui définit, en principe, l'intertextualité d'une manière beaucoup plus vaste que je ne le fais ici et extensive en apparence à tout ce que je nomme * transtextualité [...]. [...]

De l'opposition déjà marquée entre * hypertextualité et intertextualité, je ne veux insister ici que sur ce point, limite mais décisif: contrairement à l'intertextualité telle que le décrit bien Riffaterre, le recours à $l^{\prime *}$ hypotexte n'est jamais indispensable à la simple intelligence de $l^{* *}$ hypertexte.»

Gérard Genette: Palimpsestes. La littêrature au second degré. Coll. «Poétique», Paris. Seuil. 1982. p. 8 et 450 .

> > Sur ce terme, important il va sans dire, existe l'article fouillé de Marc Angenot: "L' «intertextualité»: enquête sur l'émergence et la diffusion diun champ notionnel». Revue des sciences humaines, Lille, tome LX, no 189, janvier-mars 1983, p. 121-135.

\section{Infra-biblio-intertexte}

RÉF. — Jean Ricardou, 1982

DÉF. - «Pour le mélange, en révérence, l'attache est de l'ordre d'une pressante signature. C'est parce que, en son paraphe, il en assume la paternité que le signataire est reconnu capable de réunir et de subsumer les fragments disparates du sien recueil. Ou, si l'on préfère, ce qui assure en loccurrence le ciment du divers, c'est. hors les textes, une concordance imaginaire. La mise ensemble de ces écrits semble légitime en ce que, issus chacun de même plume, ils sont admis pour l'expression d'une confortable fantasmagorique identité: l'auteur. Bref, du moins en tant que pure accumulation de 
pièces brèves selon un seul livre, le mélange a une structure d'infrabiblio-intertexte.»

Jean Ricardou: Le théâtre des métamorphoses. Coll. «Fiction \& Cie», Paris. Seuil. 1982. p. 280.

\section{Ultra-biblio-intertexte}

RÉF. — Jean Ricardou, 1982

DÉF. — «Pour le * mixte, en revanche, la ligature est le résultat d'une précise tâche. C'est parce que, selon des agrafes, il en assure la connexité, que le scripteur est en mesure de réunir et d'articuler les fragments disparates dudit recueil. Ou, si l'on aime mieux: ce qui assure ici le ciment du divers, c'est, entre les textes, des correspondances tissulaires. [...] Bref, du moins en tant qu'inventive élaboration de pièces diverses entretissées dans un seul livre, le * mixte a une structure d'ultra-biblio-intertexte."

Jean Ricardou: Le théatre des métamorphoses. Coll. «Fiction \& Cie», Paris, Seuil, 1982, p. 280-281.

EX. — Le livre ici cité, faut-il le rappeler, est ce * milletexte (ou mixte).

\section{Graphotexte}

RÉF. — Jean Ricardou, 1978

DÉF. - «En effet, l'* intertexte général, ou ensemble de tous les textes, se subdivise, opératoirement, en plusieurs espèces d'* intertextes restreints. Entre autres, ce que nous pourrons nommer graphotexte, ou ensemble des textes assumés par le même signataire |... I.»

Jean Ricardou: Nouveaux problèmes du roman. Coll. «Poétique», Paris. Seuil, 1978, p. 303.

\section{Anthologicotexte}

RÉF. — Jean Ricardou, 1978

DÉF. - «En effet, l** intertexte général, ou ensemble de tous les 
textes, se subdivise, opératoirement, en plusieurs espèces $d^{\prime *}$ intertextes restreints. Entre autres, ce que nous pourrons nommer $[. .$. anthologicotexte, ou ensemble des textes de signataires différents réunis dans un même livre [...]."

Jean Ricardou: Nouveaux problèmes du roman. Coll. «Poétique», Paris. Seuil. 1978. p. 303-304.

\section{Bibliotexte}

RÉF. — Jean Ricardou, 1978

DÉF. - «En effet, l'* intertexte général, ou ensemble de tous les textes, se subdivise, opératoirement, en plusieurs espèces $d^{* *}$ intertextes restreints. Entre autres, ce que nous pourrons nommer [...] bibliotexte, ou ensemble des textes évoqués dans un texte $(. .) .$. Jean Ricardou: Nouveaux problèmes du roman. Coll. «Poétique», Paris. Seuil. 1978, p. 303-304.

\section{Infra-inter-bibliotexte}

RÉF. - Jean Ricardou, 1982

DÉF. - «[...] cette réunion de brefs écrits variés sous l'autorité d'un nom, et qu'on appelle un mélange, n'est rien de moins qu'une mise en abyme de cette réunion de longs écrits divers sous le nom d'un auteur, et qu'on intitule une oeuvre. Bref, du moins en tant que sûre collection de pièces amples selon une série de livres, l'oeuvre a une structure d'infra-inter-bibliotexte."

Jean Ricardou: Le théâtre des métamorphoses. Coll. «Fiction $\varepsilon$ Cie», Paris, Seuil, 1982, p. 280.

\section{Ultra-inter-bibliotexte}

RÉF. - Jean Ricardou, $19 \dot{9} 82$

DÉF. - «[... cette réunion de brefs écrits divers (...) et quion appelle un * mixte, n'est rien moins qu'une mise en abyme de cette réunion de longs écrits variés (...) et qu'on pourrait nommer une pieuvre. Bref, du moins en tant qu'attentive fabrication de pièces amples selon une série de livres, la pieuvre a une structure d'ultra-inter-bibliotexte." 
Jean Ricardou: Le théâtre des métamorphoses. Coll. "Fiction E Cie», Paris. Seuil, 1982, p. 281.

\section{Syntexte}

RÉF. - Jean Ricardou, 1978

DÉF. - "De la même manière, nous appellerions syntexte l’* intertexte restreint composé de l'ensemble des textes dont chacun, sans appartenir à aucune des catégories précédentes $~_{*}^{*}$ graphotexte, * anthologicotexte, * bibliotexte], entretient un nombre remarquable de rapports.avec le texte en cause.»

Jean Ricardou: Nouveaux problèmes du roman. Coll. «Poétique», Paris, Seuil, 1978, p. 304 (voir aussi p. 129).

\section{Péritexte}

RÉF. - Jean Ricardou, 1975; Jan Baetens, 1983; Gérard Genette, 1987

DÉF. - " $L$ '* intertextualité restreinte, ce n'est pas seulement, comme on a pu le croire, l'ensemble des textes subsumé par une même signature. L*intertextualité restreinte, cela peut être aussi ce que j'ai appelé ailleurs le péri-texte ou la bibliothèque du texte. Tel texte en fait partie si, et seulement si, il est lié au texte étudié par un groupe de relations et non pas une seule.»

Jean Ricardou: Colloque Robbe-Grillet Itenu à Cerisy en 1975|, tome I. Coll. « 10/18", no 1079, Paris, UGE, 1976, p. 166.

\> Cette définition sera abandonnée par l'auteur lui-même dans le cadre de réaménagements terminologiques: voir désormais * syntexte et * bibliotexte.

$\S$ «Pour nommer les éléments entourant l'écrit, nous disposons actuellement d'au moins deux termes: * paratexte (Genette, (Palimpsestes)) et périgraphie (Compagnon, La seconde main [Seuil, coll. «Poétique», 1979]).

[...] Dans Palimpsestes, "para» reçoit la justification suivante: «ll faut l'entendre au sens ambigu, voire hypocrite, qui fonctionne dans des adjectifs comme parafiscal ou paramilitaire» (p.9). Le prestige de son auteur aidant, "para" semble condamné à s'imposer, mais pour une double raison, "péri» doit lui être préféré. Tout en accen- 
tuant fort utilement la topologie du livre, ce préfixe élude les connotations péjoratives que charrie "para" (le * paratexte serait au texte ce que la paralittérature est à la littérature). En dautres termes, "péri» dit l'insularité de l'écrit/texte, sans déprécier les zones qui l'entourent." Jan Baetens: «Bande à part? (sur la pratique de la bande-annonce)". Conséquences. Paris. no. 1, automne 1983, p. 67.

>> > J. Baetens discute ici à partir de Palimpsestes (1982) et d'une entrevue de G. Genette publiée dans Le monde dimanche (5 juin 1983, p. XIV). Dans Seuils (1987), G. Genette subsume sous le terme de * paratexte autant le péritexte que l* épitexte: "Comme il doit désormais aller de soi, péritexte et * épitexte se partagent exhaustivement et sans reste le champ spatial du * paratexte; autrement dit, pour les amateurs de formules, " paratexte $=$ péritexte + "épitexte" (p. 11).

+ «Un élément du * paratexte, si du moins il consiste en un message matérialisé, a nécessairement un emplacement, que l'on peut situer par rapport à celui du texte lui-même: autour du texte, dans l'espace du même volume, comme le titre ou la préface, et parfois inséré dans les interstices du texte, comme les titres de chapitres ou certaines notes; jappellerai péritexte cette première catégorie spatiale, certainement la plus typique et dont traiteront nos onze premiers chapitres.

[...] J'appelle péritexte éditorial toute cette zone du péritexte qui se trouve sous la responsabilité directe et principale (mais non exclusive) de l'éditeur, ou peut-être, plus abstraitement mais plus exactement, de l'édition, c'est-à-dire du fait qu'un livre est édité et éventuellement réédité, et proposé au public sous une ou plusieurs présentations plus ou moins diverses. Le mot zone indique que le trait caractéristique de cet aspect du * paratexte est essentiellement spatial et matériel; il s'agit du péritexte le plus extérieur: la couverture, la page de titre et leurs annexes; et de la réalisation matérielle du livre, dont l'exécution relève de l'imprimeur, mais la décision, de l'éditeur, en concertation éventuelle avec l'auteur: choix du format, du papier, de la composition typographique, etc."

Gérard Genette: Seuils. Coll. “Poétique». Paris. Seuil, 1987. p. 10 et 20.

EX. - Les 4 premiers chapitres (Seuils, p. 20-109) portent sur le péritexte éditorial. Les 7 chapitres suivants (p. 110-315), portent, sauf erreur (?), sur l'autre zone du péritexte.

\section{Hypotexte}

RÉF. - Mieke Bal, 1979; Gérard Genette, 1982 
> > Dans "Notes on Narrative Embedding" (communication à un symposium international, Tel Aviv et Jérusalem, juin 1979], article publié dans Poetics Today, Jérusalem, vol. 2, no 2, 1981, p. 41-59, Mieke Bal rappelle (p. 43) que dans son livre intitulé Narratologie. Essais sur la signification narrative dans quatre romans modernes (Paris, Klincksieck, 1977, p. 35), elle suggérait déjà de remplacer par hypo- le méta- du récit métadiégétique dont parle G. Genette dans "Discours du récit» (Figures III. Coll. "Poétique», Paris, Seuil, 1972, p. 238-239 par exemple). Dans Palimpsestes, G. Genette confirmera (p. 11) que l'hypotexte selon M. Bal est bien (c'est-à-dire à peu près) le récit métadiégétique selon "Discours du récit», réservant le terme hypotexte à la relation duelle hypotexte/* hypertexte dans laquelle il prend une tout autre signification.

$\S$ «[Le quatrième type de * transtextualité est l']*'hypertextualité. J'entends par là toute relation unissant un texte $B$ (que j'appellerai * hypertexte) à un texte antérieur $A$ (que j'appellerai, bien sûr, hypotexte) sur lequel il se greffe d'une manière qui n'est pas celle du commentaire."

Gérard Genette: Palimpsestes. La littérature au second degré. Coll. «Poétique», Paris, Seuil, 1982, p. 11-12.

EX. - Le livre ici cité, à peu près dans son entier.

> > G. Genette (Palimpsestes, p. 77) parle d'un «récit tuteur (...) déjà lui-même pluritextuel» et donne comme exemple un hypotexte actualisé quatre fois (dans le texte de chacun des Evangiles) et qui sert de «tuteur» à un seul * hypertexte.

\section{Hypertexte, hypertextualité}

RÉF. - Gérard Genette, 1982

DÉF. - «J'appelle donc hypertexte tout texte dérivé d'une texte antérieur [ ${ }^{*}$ hypotexte) par transformation simple (nous dirons désormais transformation tout court) ou par transformation indirecte: nous dirons imitation. (...)

[...] l'hypertextualité est une pratique transgénérique qui comprend quelques genres dits "mineurs» comme la parodie, le travestissement, le pastiche, le digest, etc., et qui traverse tous les autres.

[...] l'hypertextualité est évidemment [...] un des traits par lesquels une certaine modernité, ou postmodernité, renoue avec une tradition «prémoderne»... .

[...] I'hypertexte est presque toujours fictionnel, fiction dérivée d'une 
autre fiction, ou d'un récit d'événements réels. C'est une donnée de fait, d'ailleurs, et non de droit: l'hypertexte peut être non fictionnel, en particulier lorsqu'il dérive d'une oeuvre elle-même non fictionnelle. [...] Le * métatexte, lui, est non fictionnel par essence. D'autre part, nous l'avons constamment observé, l'hypertexte a toujours peu ou prou valeur de ${ }^{*}$ métatexte [...]. L'hypertexte est donc à bien des égards, en termes aristotéliciens, plus puissant que le * métatexte: plus libre de ses allures, il le déborde sans réciproque.

[...] Tout hypertexte, fût-ce un pastiche, peut, sans "agrammaticalité»

( $M$. Riffaterre) perceptible, se lire pour lui-même, et comporte une signification autonome, et donc, d'une certaine manière, suffisante. Mais suffisante ne signifie pas exhaustive. (...) un hypertexte peut à la fois se lire pour lui-même, et dans sa relation à son * hypotexte. [...] L'hypertexte gagne donc toujours - même si ce gain peut être jugé, comme on dit de certaines grandeurs, négatif - à la perception de son être hypertextuel.»

Gérard Genette: Palimpsestes. La littérature au second degré. Coll. «Poétique», Paris, Seuil. 1982, p. 14 et $448-451$.

>> La p. 37 de Palimpsestes contient l'important «Tableau général des pratiques hypertextuelles".

EX. - Bernard Brugière: «De la parodie à une esthétique de la duplication: étude de Travesties de Tom Stoppard". Etudes anglaises, Paris, vol. XXXVI, no 2-3, 1983, p. 267-280.

\section{Contretexte}

RÉF. - Pierre Bec, 1984

DÉF. — Le contre-texte «n'est pas ambigu. Il s installe en effet dans le code littéraire, utilise ses procédés jusqu'à l'exaspération, mais le dévie fondamentalement de son contenu référentiel. II n'y a donc pas d'ambiguité à proprement parler, mais juxtaposition concertée, à des fins ludiques et burlesques, d'un code littéraire donné et d'un contenu marginal, voire subversif. Le code textuel endémique reste donc bien l'indispensable référence, fonctionne toujours dans la plénitude de ses moyens, mais à contre-courant. C'est dans cet effet de distorsion, non ambigü puisque voulu et ressenti comme tel, que se situe la véhémence séditieuse du contre-texte dans ses registres les plus divers: parodique, burlesque, humoristique, scatologique et obscène; registres qui se mêlent souvent d'ailleurs, cumulant ainsi leurs effets. $|\ldots|$ 
Le contre-texte est donc, par définition, un texte minoritaire et marginalisé, une sorte d'infra-littérature (underground). Sa référence paradigmatique reste le texte, dont il se démarque, et son récepteur, inévitablement, le mème que celui du texte. Car sa réception et son impact sont étroitement liés aux modalités du code textuel majoritaire. (...)

[...] Il n'est donc pas étonnant que la thématique fondamentale du contre-texte gravite présque toujours, tout comme le texte luimême autour de la fin amor. Avec, évidemment, une finalité délibérée de marginalisation, de négation ou de distorsion concertée. On assiste donc - et toute proportion gardée - à une sorte de carnavalisation de la littérature qui redonne au texte une gaillardise ( $P$. Zumthor parle de turgescence), à la fois thérapeutique et destructrice de l'ordre établi. (...)

[...] Ajoutons enfin qu'un même troubadour peut parfaitement assumer à lui tout seul la dialectique du texte (registres de la fin amor, de la morale, de la politique, du didactisme, etc.) et du contretexte (burlesque, scatologique, obscène, etc.).»

Pierre Bec (édition bilingue présenté par): Burlesque et obscénité chez les troubadours. Le contre-texte au Moyen âge, série "Moyen âge», Paris, Stock, 1984. p. 11, 13. 14 et 20.

>> > Le texte et le contretexte ainsi définis renvoyent à l'* hypotexte et à l'* hypertexte selon Genette et, dans les pratiques hypertextuelles, plus particulièrement au travestissement burlesque et au pastiche (voir Palimpsestes, p. 64-92 ainsi que le tableau de la p. 161).

EX. - Le livre ici cité.

\section{Mimotexte, mimotextualité}

\section{RÉF. - Gérard Genette, 1982}

DÉF. - «[...] mimétisme. J'appellerai donc ainsi, en amont de la distinction de régime entre pastiche, charge et forgerie, tout trait ponctuel d'imitation; et (pendant que j'y suis) mimotexte tout texte imitatif, ou agencement de mimétismes.

(...) L'essence du mimotexte, son trait spécifique nécessaire et suffisant, est l'imitation d'un style: il y a pastiche (ou charge, ou forgerie) quand un texte manifeste, en l'effectuant, l'imitation d'un style. [...] il est impossible d'imiter directement un texte, on ne peut l'imiter qu'indirectement en pratiquant son style dans un autre texte." Gérard Genette: Palimpsestes. La littérature au second degré. Coll. «Poétique». Paris. Seuil, 1982, p. 87-91. 
EX. - Les chapitres XIV à XXXIX (p. 80-236) du livre ici cité.

\section{Intermimotexte, intermimotextualité}

RÉF. - Daniel Bilous, 1983

DÉF. - «Si l'on qualifie de mimotextuels les rapports verticaux entre les * mimotextes et leurs cibles, on dira inter- et/ou hypermimotextuels ceux qui, horizontalement, unissent les * mimotextes entre eux.

$\lceil$... Deux pastiches macrosimilaires tissent des liens d'intermimotextualité."

Daniel Bilous: «Intertexte/pastiche. L'intermimotexte». Texte, Toronto, no 2 (no intitulé Lintertextualité, Intertexte, autotexte, intratexte), 1983, p. 151 et 156.

>> > Voir le schéma de la p. 151 où, en effet, sont disposés verticalement et horizontalement les termes.

EX. - L'article ici cité analyse un intermimotexte S. Mallarmé/P. Valéry. 